\title{
Characteristics and Clinical Implications of Carbapenemase- Producing Klebsiella pneumoniae Colonization and Infection, Italy
}

Marianna Rossi, Liliane Chatenoud, Floriana Gona, Isabella Sala, Giovanni Nattino, Alessia D’Antonio, Daniele Castelli, Teresa Itri, Paola Morelli, Sara Bigoni, Chiara Aldieri, Roberto Martegani, Paolo A. Grossi, Cecilia Del Curto, Stefania Piconi, Sara G. Rimoldi, Paola Brambilla, Paolo Bonfanti, Evelyn Van Hauwermeiren, Massimo Puoti, Gianni Gattuso, Chiara Cerri, Mario C. Raviglione, Daniela M. Cirillo, Alessandra Bandera, Andrea Gori; The KPC-Kp Study Group ${ }^{1}$

\section{Medscape ACTIVITY}

In support of improving patient care, this activity has been planned and implemented by Medscape, LLC and Emerging Infectious Diseases. Medscape, LLC is jointly accredited by the Accreditation Council for Continuing Medical Education (ACCME), the Accreditation Council for Pharmacy Education (ACPE), and the American Nurses Credentialing Center (ANCC), to provide continuing education for the healthcare team

Medscape, LLC designates this Journal-based CME activity for a maximum of 1.00 AMA PRA Category 1 Credit(s) ${ }^{\mathrm{TM}}$. Physicians should claim only the credit commensurate with the extent of their participation in the activity.

Successful completion of this CME activity, which includes participation in the evaluation component, enables the participant to earn up to 1.0 MOC points in the American Board of Internal Medicine's (ABIM) Maintenance of Certification (MOC) program. Participants will earn MOC points equivalent to the amount of CME credits claimed for the activity. It is the CME activity provider's responsibility to submit participant completion information to ACCME for the purpose of granting ABIM MOC credit.

All other clinicians completing this activity will be issued a certificate of participation. To participate in this journal CME activity: (1) review the learning objectives and author disclosures; (2) study the education content; (3) take the post-test with a 75\% minimum passing score and complete the evaluation at http://www.medscape.org/journal/eid; and (4) view/print certificate. For CME questions, see page 1550.

Release date: May 22, 2021; Expiration date: May 22, 2022

Learning Objectives

Upon completion of this activity, participants will be able to:

- Describe epidemiology of KPC-Kp and molecular characterization of KPC-Kp strains in colonized and infected inpatients with mild (MI) or serious (SI) infections in Italy, according to a multicenter cohort study of 1,071 patients with KPC-Kp

- Determine clinical characteristics and outcomes of KPC-Kp in colonized and infected inpatients with MI or SI in Italy, according to a multicenter cohort study

- Identify treatment and other clinical implications of KPC-Kp in colonized and infected inpatients with MI or SI in Italy, according to a multicenter cohort study

CME Editor

Amy J. Guinn, BA, MA, Copyeditor, Emerging Infectious Diseases. Disclosure: Amy J. Guinn, BA, MA, has disclosed no relevant financial relationships.

\section{CME Author}

Laurie Barclay, MD, freelance writer and reviewer, Medscape, LLC. Disclosure: Laurie Barclay, MD, has disclosed no relevant financial relationships.

Authors

Disclosures: Disclosures: Marianna Rossi, MD, PhD; Liliane Chatenoud, PhD; Floriana Gona, PhD; Isabella Sala, MS; Giovanni Nattino, PhD; Alessia D'Antonio, MD; Daniele Castelli, MS; Teresa Itri, MSc; Paola Morelli, PhD; Sara Bigoni, MD; Chiara Aldieri, MD; Roberto Martegani, PhD; Paolo A. Grossi, MD, PhD; Cecilia Del Curto, MD; Stefania Piconi, MD; Sara G. Rimoldi, MS; Paola Brambilla, PhD; Evelyn Van Hauwermeiren, MD; Gianni Gattuso, MD; Chiara Cerri, MD; Mario C. Raviglione, MD; Alessandra Bandera, MD, PhD; and Andrea Gori, MD, have disclosed no relevant financial relationships. Paolo Bonfanti, MD, has disclosed the following relevant financial relationships: served as an advisor or consultant for Janssen-Cilag; Gilead Sciences, Inc.; ViiV Healthcare; served as a speaker or a member of a speakers bureau for Gilead Sciences, Inc.; Pfizer Inc. Massimo Puoti, MD, has disclosed the following relevant financial relationships: served as an advisor or consultant for AbbVie Inc.; Merck \& Co., Inc.; served as a speaker or a member of a speakers bureau for AbbVie Inc.; Gilead Sciences, Inc.; Merck \& Co., Inc.; received grants for clinical research from AbbVie Inc.; Eli Lilly and Company; Gilead Sciences, Inc. Daniela M. Cirillo, MD, PhD, has disclosed the following relevant financial relationships: served as an advisor or consultant for bioMérieux; received grants for clinical research from bioMérieux; DiaSorin. 
Klebsiella pneumoniae carbapenemase-producing $K$. pneumoniae (KPC-Kp) has been endemic in Italy since 2013. In a multicenter cohort study, we investigated various aspects of KPC-Kp among patients, including 15-day mortality rates and delays in adequate therapy. Most (77\%) KPC-Kp strains were sequence types ST512 or ST307. During 2017, KPC-Kp prevalence was 3.26 cases $/ 1,000$ hospitalized patients. Cumulative incidence of KPC-Kp acquired $>48$ hours after hospital admission was $0.68 \%$ but varied widely between centers. Among patients with mild infections and noninfected colonized patients, 15-day mortality rates were comparable, but rates were much higher among patients with severe infections. Delays of $\geq 4$ days in receiving adequate therapy more frequently occurred among patients with mild infections than those with severe infections, and delays were less common for patients with known previous KPC-Kp colonization. Italy urgently needs a concerted surveillance system to control the spread of KPC-Kp.

$\mathrm{T}$ he global emergence and spread of carbapenem-resistant Enterobacteriaceae (CRE) pose a major health threat, causing severe illness and high healthcare costs (1). Infections caused by CRE also are associated with high mortality rates because extensive resistance to so-called last-line antimicrobial drugs, such as carbapenems, limit the treatment options (2-5). Only a few antimicrobial drugs, such as colistin, fosfomycin, tigecycline, and ceftazidime/ avibactam, are effective against CRE. Moreover, the remaining therapeutic options often have high toxicity profiles, and rates of resistance to these antimicrobial drugs already are increasing (6).

In a 2014 study conducted by the European Survey of Carbapenemase-Producing Enterobacteriaceae (EuSCAPE) Working Group, 455 sentinel hospitals in 36 countries submitted clinical isolates (7). Among the 2,703 isolates submitted, 2,301 (85\%) were Klebsiella pneumoniae and 402 (15\%) were Escherichia coli, including samples identified as carbapenemase producers among 850 (37\%) K. pneumoniae and $77(19 \%)$ E. coli isolates. Identified carbapenemaseproducers included 4 gene families: K. pneumoniae

Author affiliations: S. Gerardo Hospital, Monza, Italy (M. Rossi,

T. Itri, D. Castelli, P. Bonfanti); Istituto di Ricerche Farmacologiche Mario Negri IRCCS, Milan, Italy (L. Chatenoud, I. Sala, G. Nattino, A. D'Antonio); IRCCS San Raffaele Scientific Institute, Milan (F. Gona, C. Del Curto, D.M. Cirillo); Fondazione IRCCS Ca' Granda, Ospedale Maggiore Policlinico, Milan (T. Itri, A. Bandera, A. Gori); Humanitas University, Milan (P. Morelli); Papa Giovanni XXIII Hospital, Bergamo, Italy (S. Bigoni); University of Milan San Paolo Hospital, Milan (C. Aldieri); Busto Arsizio Hospital, Busto Arsizio, Italy (R. Martegani); University of Insubria, ASST Sette carbapenemase (KPC), New Delhi metallo- $\beta$ lactamase, oxacillinase 48-like, and Verona integronencoded metallo- $\beta$-lactamase (7). Positive clinical specimens were found in 1.3 patients $/ 10,000$ hospital admissions, but prevalence differed greatly between countries and the highest rates were registered in countries in the Mediterranean and Balkan regions (7). Among these countries, Italy, Greece, and Romania reported the highest percentages of carbapenem resistance. In addition, CRE rates increased from 15\% in 2010 to $36 \%$ in $2016(8-10)$, and CRE became endemic in Greece in 2010 and Italy in 2013 (11). Nevertheless, currently published information is too scant to define the complete picture of KPC K. pneumoniae $(\mathrm{KPC}-\mathrm{K} p$ ) epidemiology in both clinical isolates and surveillance screening samples (12).

In this context, we set up a network of 15 hospitals in Lombardy, the most populous region in Italy, and established a cohort of patients affected by $\mathrm{KCP}-\mathrm{K} p$. The overarching goal of the KPC-Kp Study Group was to identify the challenges of controlling the spread of the bacterium. We describe KPC-Kp epidemiology, treatment, and in-hospital mortality rates, along with molecular characterization of KPC$K p$ strains in colonized and infected inpatients.

\section{Methods}

\section{Study Design, Setting, and Patients}

We conducted a multicenter cohort study during June 2016-April 2018, which included 15 hospitals in Lombardy (Figure 1; Appendix, https://wwwnc. cdc.gov/EID/article/27/5/20-3662-App1.pdf). We asked each enrolled hospital to include data on all consecutively hospitalized adult patients who had $\geq 1$ positive KPC- $\mathrm{K} p$ isolate during their hospital stay. For patients hospitalized multiple times during the study period, we only considered the first hospitalization. For centers including patients during 2017, the year for which we had a full 12 months of data, we retrieved the administrative datasets of all admitted patients (Figure 1). We merged these data with

Laghi-Varese, Italy (P.A. Grossi); ASST Fatebenefratelli Sacco, Milan (S. Piconi, S.G. Rimoldi); Clinic of Infectious Diseases of Istituti Ospedalieri of Cremona, Cremona, Italy (P. Brambilla); University of Milan-Bicocca, Milan (P. Bonfanti); University of Brescia and ASST Spedali Civili, Brescia, Italy (E. Van Hauwermeiren); ASST Grande Ospedale Metropolitano Niguarda, Milan (M. Puoti); Carlo Poma Hospital, Mantova, Italy (G. Gattuso); Hospital of Lodi, Lodi, Italy (C. Cerri); University of Milan, Milan (M.C. Raviglione, A. Bandera, A. Gori)

DOI: https://doi.org/10.3201/eid2705.203662 


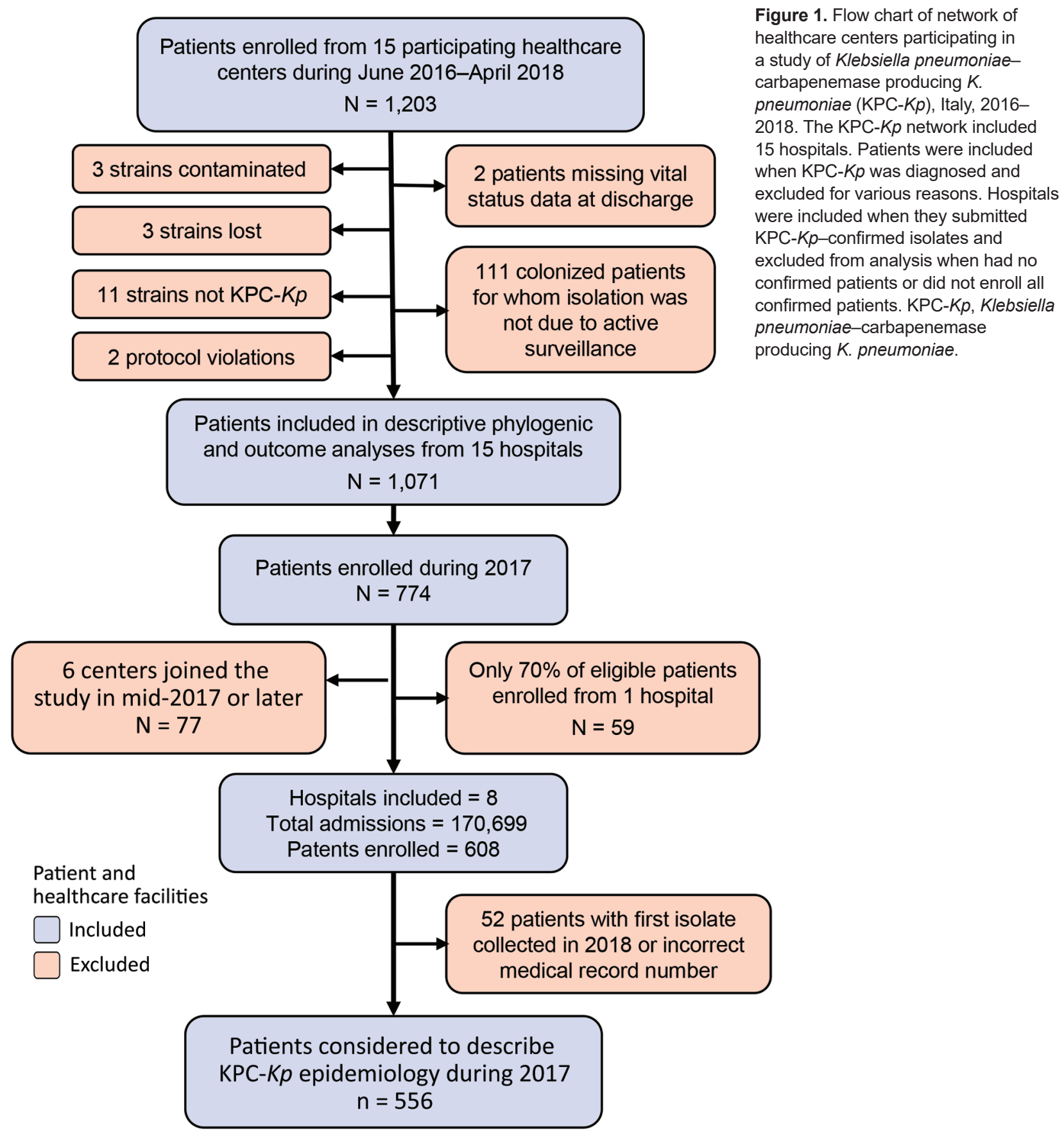

those available in the KPC-Kp patient cohort database and used the combined dataset to describe KPC-Kp epidemiology in the hospitalized population.

The study protocol was first approved by the Research Ethics Committee of the coordinating center, Ospedale San Gerardo (Monza, Italy). Informed consent requirement was waived due to the study's observational, noninterventional design. The study protocol was subsequently approved by the ethics committees of the 14 other participating centers. In accordance with local ethics committee requirements, 3 centers did not waive informed consent. Because this was an observational study, treatment for KPC$K p$ infections was at the discretion of the attending physicians and no change to the center-specific surveillance protocol was required.

In all centers, intensive care unit (ICU) patients were tested for CRE at admission and weekly through rectal swab specimens or other surveillance cultures. The same protocol was applied heterogeneously in 
hospital wards in which patients are considered to be at higher risk of acquiring CRE, such as hematology, solid organ transplant, and geriatric units (Appendix Table 1). For the other wards, most centers performed surveillance rectal swab specimens at admission on the basis of major risk factors for CRE, such as previous CRE colonization, previous hospitalization during the 12 months before inclusion, or both. Of note, only 3 of the 15 participating centers, B, C, and I (Appendix Table 1), combined the 2 surveillance strategies described for specific wards and patients at higher risk of acquiring $C R E$.

\section{Patient Classification}

Patients were classified according to the most clinically relevant KPC-Kp isolate collected from them between hospital admission and discharge. Thus, for patients whose first isolate was attributable to colonization and a subsequent isolate was attributed to an infection, only the second isolate was considered. We used US Centers for Disease Control and Prevention criteria (13) to define diagnosed infection and diagnosis was confirmed by an infectious disease specialist. Infections were classified as KPC-Kp bacteremia when a blood culture was positive for a KPC-Kp strain with or without KPC- K $p$-positive cultures from $\geq 1$ other site and the patient had clinical signs of systemic inflammatory response syndrome requiring antimicrobial drug treatment. We defined nonbacteremic KPC-Kp infections by documented recovery of a KPC- Kp isolate from nonblood cultures, such as intra-abdominal wounds, urine, or bronchoalveolar lavage fluid; absence of KPC-Kppositive blood culture during the index hospitalization; and clinical signs of infection.

In line with other studies (14), we classified KPC$K p$ cases according to infection severity. We classified cases of KPC-Kp bloodstream or lower respiratory tract infections, and clinical presentation of septic shock, regardless of infection site, as severe infections. We classified infections from the urinary tract, surgical wounds, or other sites without septic shock as mild infections. We classified all cases identified through active surveillance as colonized when $\geq 1$ culture sample grew KPC-Kp but the patient did not develop KPC-Kp infection during hospitalization.

\section{Data Collection}

For patients included in the KPC-Kp cohort, data were entered into the web-based case form after pseudonymization of personal data. Data were collected on demographic characteristics, medical history, underlying diseases, previous hospitalization, previous
KPC- $K p$ infection, surgery $\leq 30$ days before KPC-Kp isolation, invasive procedures $\leq 72$ hours before KPC$K p$ isolation, antimicrobial drug therapy $\leq 30$ days before KPC-Kp isolation, dates of admission to hospital, and ward of isolation. Date of hospital discharge and patient status at discharge also were collected. The date and ward where the patient was hospitalized when KPC-Kp was isolated, the source of isolation, and resistance spectrum also were collected and entered into the web-based case record form. Antimicrobial treatment, including empirical treatment and post-antibiogram treatment regimen, were recorded. Empirical treatment was defined as adequate when it included $\geq 1$ antimicrobial drug with in vitro activity against the KPC-Kp isolate. Data were collected in a web-based case report form.

For enrolled centers submitting patient data during 2017, we retrieved the clinical record datasets of all admitted patients after pseudonymization of personal information. To verify centers included all eligible patients, we retrieved the total number of patients with $\geq 1 \mathrm{KPC}-\mathrm{Kp}$-positive isolate registered in the microbiology laboratory of each center and compared that with the total number of patients included in the cohort (Appendix).

\section{Microbiology and Genomic Analysis}

The clinical microbiology laboratory of each of the 15 participating centers performed isolate identification and routine antimicrobial susceptibility testing (Appendix). CRE was defined by using Clinical and Laboratory Standard Institute guidelines (15). All bacterial strains were sent to a central microbiological laboratory at Ospedale San Raffaele for whole-genome sequencing (Appendix).

\section{Statistical Analysis}

We estimated the prevalence of KPC-Kp in hospitalized patients in the region of Lombardy during 2017, the cumulative incidence of acquired KPC-Kp infections among hospitalized patients, and the cumulative incidence of acquired KPC- $K p$ infections occurring $>48$ hours after hospital admission among hospitalized patients in the same region. We calculated and reported crude estimates for all centers and estimates standardized by age and ward of isolation (Appendix).

To study the role of KPC-Kp infection severity on 15-day mortality rates, we considered a multivariable Cox proportional hazard model and the related hazard ratio (HR) estimates and adjusted by center for a random effect and number of days from hospitalization to KPC-Kp isolation. Colonized patients 
frequently have shorter hospital stays than infected patients. Because a shorter discharge time could affect our results, we performed a sensitivity analysis in which we excluded early-discharge patients. We performed a subgroup analysis to quantify excess mortality hazard due to septic shock among patients with bloodstream infections (Appendix).

We used multivariable mixed logistic regression models and accounted for clustering at the center level to evaluate the association between patient characteristics and delayed or inadequate empirical therapy, which we considered as outcome variables. We adjusted the models for age and type of KPC-Kp infection.

\section{Results}

\section{Center Characteristics}

Among all centers, the median number of annual admissions was 27,600 (interquartile range [IQR] 18,287-40,000). Among 15 enrolled centers, 9 (60\%) maintained enrollment over 12 consecutive months; centers had a mean enrollment duration of 13.8 months (Appendix Figure 1).

\section{Patient Baseline Characteristics}

Among 1,203 consecutive KPC-Kp-positive hospitalized patients found during study, $89.0 \%(1,071)$ were considered in the analyses and 11\% (132) were excluded for various reasons (Figure 1).

The median age among patients was 72 (IQR $61-80$ ) years, $65 \%$ were male, and $35 \%$ were female; KPC-Kp was isolated from 275 (25.7\%) ICU patients (Table 1). Among patients in the study cohort, $>90 \%$ had $\geq 1$ underlying condition, $40 \%$ of whom had congestive heart failure, peripheral vascular disease, or chronic renal failure. Severe infections were diagnosed in $221(20 \%)$ patients and mild infections in $109(10 \%)$ patients. Colonized patients $\mathrm{n}=741$, $69.2 \%$ ), had a median of 6 days between hospitalization and KPC-Kp isolation, which was much lower than for patients with severe (median 12 days) or mild (median 11 days) infections. Bloodstream infections accounted for $54 \%$ of all infections, and rectal swab samples accounted for $67 \%$ of all colonizations (Appendix Figure 2).

\section{Distribution, Phylogeny, and Resistance Mechanisms of KPC-Kp Clones}

Among the 1,071 patient strains isolated, 82 were from colonized patients included at the end of April 2018; these samples did not arrive at the central laboratory in time for genotyping. Of the 989 strains analyzed, 32 different sequence types (STs) were identified. The most numerous clones were ST512 in $45 \%$ (441), ST307 in 33\% (326), ST258 in 7\% (71), and ST101 in 6\% (57) of isolates (Appendix Table 3). We identified 2 KPC variants, KPC-2 and KPC-3, in 68\% of isolates. KPC-2 was absent in ST512 but predominant in ST307 and ST258. Core-genome, single-nucleotide polymorphism (SNP) analysis revealed that ST512 was scattered across all centers, but ST307 was represented in smaller, more localized clusters (Figure 2; Appendix Table 3).

\begin{tabular}{|c|c|}
\hline Characteristics & $\begin{array}{c}\text { KPC-Kp patients } \\
\mathrm{n}=1,071\end{array}$ \\
\hline \multicolumn{2}{|l|}{ Sex } \\
\hline M & $694(64.8)$ \\
\hline $\mathrm{F}$ & $377(35.2)$ \\
\hline Median age (IQR) & $72(61-80)$ \\
\hline \multicolumn{2}{|l|}{ Ward of isolation } \\
\hline Intensive care unit & $275(25.7)$ \\
\hline Infectious diseases & $81(7.6)$ \\
\hline Surgery & 149 (13.9) \\
\hline Geriatrics & $47(4.4)$ \\
\hline Oncology & $34(3.2)$ \\
\hline Hematology & $42(3.9)$ \\
\hline Other medical wards & $443(41.4)$ \\
\hline KPC-Kp colonization in previous $12 \mathrm{mo}$ & $333(31.1)$ \\
\hline Hospitalization in previous $12 \mathrm{mo}$ & $865(80.8)$ \\
\hline $\begin{array}{l}\text { Antimicrobial therapy in the } 30 \mathrm{~d} \text { before } \\
\text { hospitalization }\end{array}$ & $782(73.0)$ \\
\hline Major surgery in the previous $30 \mathrm{~d}$ & $262(24.4)$ \\
\hline Underlying conditions $†$ & $989(92.3)$ \\
\hline Congestive heart failure & $192(17.9)$ \\
\hline Peripheral vascular disease & $197(18.4)$ \\
\hline Cerebrovascular disease & $205(19.1)$ \\
\hline Chronic lung disease & $202(18.9)$ \\
\hline Chronic renal failure & $304(28.4)$ \\
\hline Cancer & $244(22.8)$ \\
\hline Diabetes & $163(15.2)$ \\
\hline Charlson index, median (IQR) & $6(4-8)$ \\
\hline Central venous catheter at isolation & $414(38.7)$ \\
\hline Urinary catheter at isolation & $562(52.5)$ \\
\hline Immunosuppressive therapy & $209(19.5)$ \\
\hline Days of hospitalization, median (IQR) & $25(14-45)$ \\
\hline \multicolumn{2}{|l|}{ KPC-Kp acquisition characteristics $\ddagger$} \\
\hline Severe infection & $221(20.6)$ \\
\hline Mild infection & 109 (10.2) \\
\hline Colonization $_{\text {sur }}$ & $741(69.2)$ \\
\hline \multicolumn{2}{|c|}{ Median time from hospitalization to isolation of strain, $\mathrm{d}(\mathrm{IQR}) \ddagger$} \\
\hline Severe infection & $12(2-22)$ \\
\hline Mild infection & $11(2-25)$ \\
\hline Colonization $_{\text {sur }}$ & $6(1-17)$ \\
\hline \multicolumn{2}{|c|}{ Median time from strain isolation to discharge or death, $d(I Q R) \ddagger$} \\
\hline Severe infection & $18(9-35)$ \\
\hline Mild infection & $20(12-35)$ \\
\hline Colonization $_{\text {sur }}$ & $13(6-22)$ \\
\hline \multicolumn{2}{|c|}{$\begin{array}{l}\text { *Values are no. (\%) except as indicated. IQR, interquartile range; KPC-Kp, } \\
\text { Klebsiella pneumoniae carbapenemase-producing Klebsiella pneumoniae. } \\
\text { †Underlying conditions and devices are listed when present in } \geq 10 \% \text { of } \\
\text { patients. } \\
\text { †Severe infection included bloodstream or lower respiratory tract infection } \\
\text { plus septic shock from other sites; Mild infection included infections from } \\
\text { other sites; and colonizedsur patients were identified through surveillance } \\
\text { protocols. }\end{array}$} \\
\hline
\end{tabular}




\section{Epidemiology of KPC-Kp}

During 2017, the estimated prevalence of KPC-Kp among hospitalized patients in the Lombardy region was 3.26 (95\% CI 2.99-3.54) per 1,000 admissions. In the same region, the overall cumulative incidence of KPC-Kp infections was $1.00 \%$ o (95\% CI $0.86 \%$ $-1.16 \%$ ) and the incidence of acquired infections occurring $>48$ hours after hospital admission was
$0.68 \%$ o (95\% CI $0.56 \%$ o- $0.82 \%$ ). The proportion of patients infected at admission, considered imported infections, was $\approx 30 \%$ in most centers. We observed marked differences across centers even after standardization by age and ward of isolation, with values ranging from $1.62 \%$ o (95\% CI $1.07 \%$ o- $2.18 \%$ o) in center A to $0.21 \%$ o $(95 \%$ CI $0.02 \%$ o- $0.40 \%$ o) in center B (Appendix Figure 3).

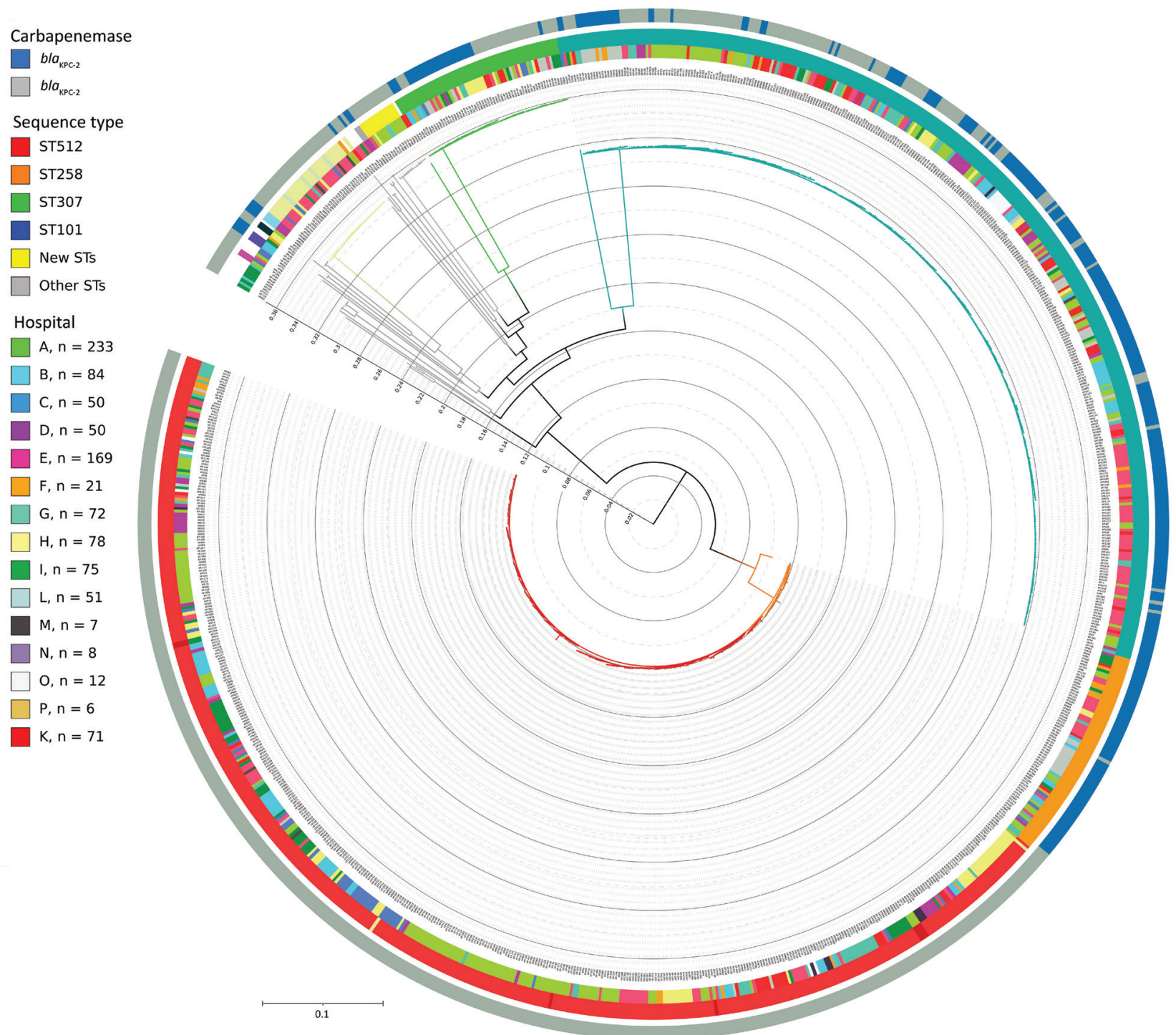

Figure 2. Phylogenetic tree of 989 Klebsiella pneumoniae genomes isolated at hospitals participating in the KPC-producing K. pneumoniae (KPC-Kp) study, Italy. The key shows the number of isolates included in the study provided by each center; 2 samples (1 from each from hospitals $A$ and I) were excluded because the total quality of the assemblies was not sufficient to have high confidence in the SNPs called through all the genome (total coverage <30). Inner circle shows the KPC-Kp mechanism identified; middle circle shows hospitals from which strains were isolated; and outer circle shows identified STs. The whole genome core single-nucleotide polymorphisms (SNPs) were extracted from the 989 K. pneumoniae genome assemblies by using kSNP3.0 (https://sourceforge. net/projects/ksnp). Parametric maximum-likelihood estimation (general time-reversible plus gamma distribution plus invariable sites) analysis with 1,000 bootstrap estimates was used to infer the phylogeny. We used IQ-TREE (http://www.iqtree.org) to generate the tree and iTOL (https://itol.embl.de) to draw the tree. Major STs are represented by branch colors; ST512 and ST307 were the predominant STs. Major branches have bootstrap values $>0.75$ for branch support. Scale bar indicates nucleotide substitutions per site. KPC, Klebsiella pneumoniae-carbapenemase; ST, sequence type. 


\section{Patient Outcomes}

In-hospital death from all causes was 34\% (95\% CI $29.2 \%-39.6 \%$ ) among KPC-Kp-infected patients and $21 \%(95 \%$ CI $17.7 \%-27.6 \%)$ among colonized patients. No differences emerged when we stratified for carbapenem-resistance mechanisms and the most prevalent clones (Appendix Table 4).

Mortality hazards (considering the first 15 days after KPC-Kp isolation), were much higher for patients with severe infection than for colonized patients, even after controlling for center, time between hospitalization and isolation, age, ward of isolation, and Charlson index (adjusted HR [aHR] $=1.93,95 \%$ CI 1.40-2.66) (Table 2). In contrast, no excess mortality hazard was noted for patients with mild infections (aHR $=0.75,95 \%$ CI 0.42-1.34) compared with colonized patients."

When we analyzed the subgroup of patients with bloodstream infections, we found clinical manifestation of septic shock more than doubled the risk for death $(\mathrm{HR}=2.71,95 \%$ CI 1.46-5.02). We found comparable results when we excluded from the analysis 343 patients discharged alive before day 15 (data not shown).

\section{Antimicrobial Drug Treatment}

On the basis of susceptibility test results, we found that $54 \%(159 / 297)$ of patients infected with KPC-Kp received adequate empirical therapy (Appendix Table 5). Empirical treatment was most frequently adequate in patients with KPC-Kp colonization during the previous 12 months and in patients with severe infection (Appendix Table 5).

Fewer treatment delays ( $<4$ days, which is considered the maximum acceptable waiting time to receive appropriate antimicrobial treatment) were reported for patients with severe KPC-Kp infection than patients with mild infections (Table 3). Patients reporting KPC-Kp colonization during the previous 12 months more frequently received prompt adequate therapy $(\mathrm{p}<0.001)$.

Among the $282 \mathrm{KPC}-\mathrm{Kp}$-infected patients treated for their infections, $62(22 \%)$ received an in vitro active drug plus carbapenem, but 29 (10\%) patients received gentamicin, fosfomycin, or tigecycline monotherapy. The most common drug combination was colistin plus tigecycline plus carbapenem, which most frequently was administered to patients with severe infections. Ceftazidime/avibactam became available in Italy in February 2018, and 26/39 (66\%) infected patients included after that date received it: 19/24 (79\%) in the severe infection group and 7/15 (47\%) in mild infection group (Appendix Table 6).

\section{Discussion}

This study provides a detailed picture of KPC-Kp burden in an endemic setting and shows that KPC-Kp poses a major challenge for Italy's healthcare system. We estimated that 1 of every 1,000 patients admitted to participating hospitals during 2017 had a positive $\mathrm{KPC}-\mathrm{Kp}$ specimen during hospitalization, which is $\approx 10$ times the estimated number of CRE infections in Europe (1.3/10,000 hospitalizations) (7). This high rate is at least partly compatible with the heterogeneity in the surveillance protocols adopted by hospitals. Another factor contributing to the high rate of KPC$\mathrm{Kp}$ could be the older age of the patient population, most of whom were men $>65$ years of age. In 2017, the median age of the adult population in Lombardy was 50 years, but the median age for the 170,699 adult patients in our study was 66 years, and $27 \%$ were $>77$ years of age. Of note, the considerable proportion of imported KPC-Kp infections, $\approx 30 \%$, for most centers, suggests that active surveillance might need to be

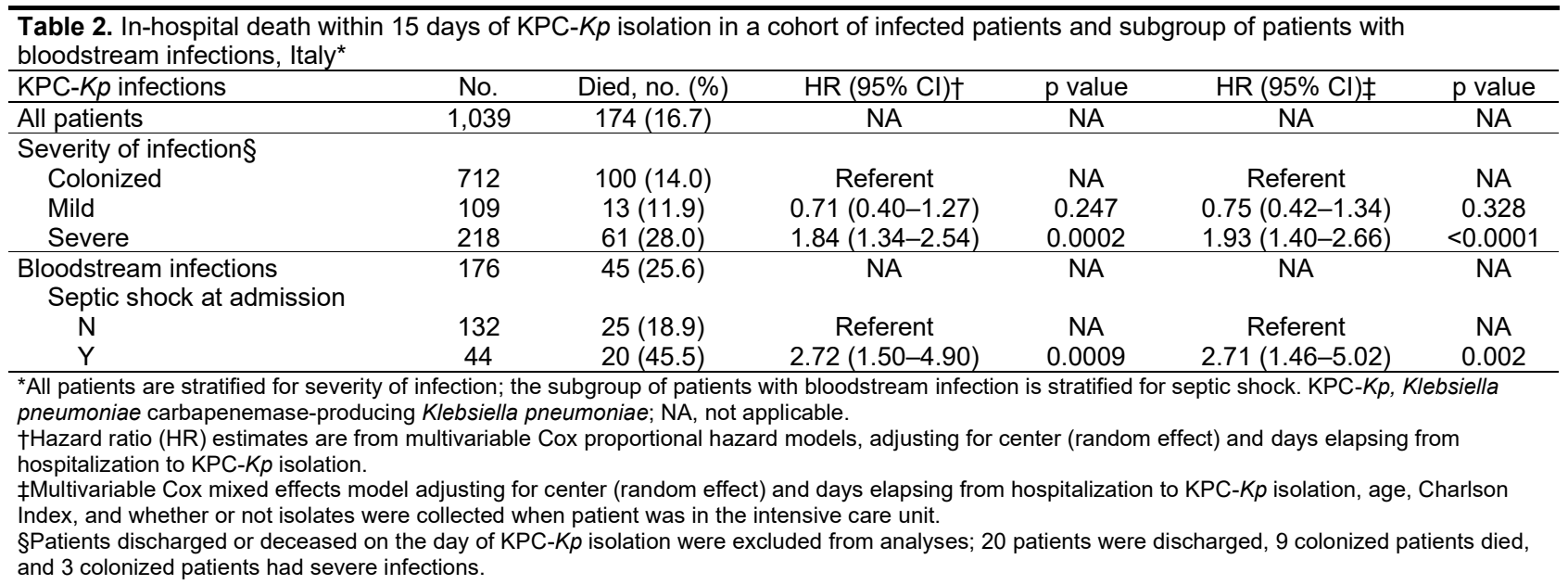


Table 3. Association between delay in receiving adequate antimicrobial therapy after KPC-Kp isolation and selected patient characteristics, Italy*

\begin{tabular}{|c|c|c|c|c|}
\hline \multirow[b]{2}{*}{ Characteristics } & \multicolumn{2}{|c|}{$\begin{array}{l}\text { Delay from KPC- } K p \text { isolation to } \\
\text { adequate antimicrobial therapy }\end{array}$} & \multirow[b]{2}{*}{$\chi^{2} \mathrm{p}$ value } & \multirow[b]{2}{*}{$\mathrm{p}$ valuet } \\
\hline & $<4 \mathrm{~d}$ & $\geq 4 \mathrm{~d}$ & & \\
\hline All & $190(63.9)$ & $107(36.0)$ & NA & NA \\
\hline Age, median (IQR) & $68.5(62-78)$ & $74(63-81)$ & 0.151 & 0.285 \\
\hline Charlson Index, median (IQR) & $5.0(4-8)$ & $6.0(4-8)$ & 0.615 & 0.439 \\
\hline \multicolumn{5}{|l|}{ Intensive care unit admission } \\
\hline Y & $41(63.1)$ & 24 (36.9) & 0.865 & 0.354 \\
\hline $\mathrm{N}$ & $149(64.2)$ & $83(35.8)$ & & \\
\hline \multicolumn{5}{|c|}{ Previous KPC-Kp colonization during the current hospitalization } \\
\hline $\mathrm{Y}$ & $46(74.2)$ & $16(25.8)$ & 0.060 & 0.118 \\
\hline $\mathrm{N}$ & $144(61.3)$ & $91(38.7)$ & & \\
\hline \multicolumn{5}{|c|}{ KPC- $K p$ colonization in the previous $12 \mathrm{mo}$} \\
\hline Y & $104(77.0)$ & $31(23.0)$ & $<0.001$ & $<0.001$ \\
\hline $\mathrm{N}$ & $86(53.2)$ & $75(46.8)$ & & \\
\hline \multicolumn{5}{|c|}{ Hospitalization in the previous $12 \mathrm{mo}$} \\
\hline Y & $149(64.5)$ & $82(35.5)$ & 0.832 & 0.779 \\
\hline $\mathrm{N}$ & $41(63.1)$ & $24(36.9)$ & & \\
\hline \multicolumn{5}{|c|}{ Antimicrobial therapy in the $30 \mathrm{~d}$ before hospitalization } \\
\hline $\mathrm{Y}$ & $145(64.0)$ & $84(36.0)$ & 0.564 & 0.627 \\
\hline $\mathrm{N}$ & $45(67.2)$ & $22(32.8)$ & & \\
\hline \multicolumn{5}{|l|}{ Major surgery $\ddagger$} \\
\hline Y & 48 (53.9) & $41(46.1)$ & 0.018 & 0.008 \\
\hline $\mathrm{N}$ & $142(74.7)$ & $66(31.7)$ & & \\
\hline \multicolumn{5}{|l|}{ KPC-Kp infection severity§ } \\
\hline Severe & $139(71.5)$ & $55(28.3)$ & 0.0002 & $<0.001$ \\
\hline Mild & $52(50.0)$ & $52(50.0)$ & & \\
\hline \multicolumn{5}{|c|}{$\begin{array}{l}\text { *Values are no. (\%) except as indicated. Delay determined according to infected patients' resistance profiles; } 33 \text { patients were excluded: } 17 \text { had follow-up } \\
\text { <3 days after isolation and } 16 \text { had no data on empirical therapies. IQR, interquartile range; KPC-Kp, Klebsiella pneumoniae-carbapenemase producing } \\
\text { Klebsiella pneumoniae; NA, not applicable. } \\
\text { †Obtained from multivariable mixed logistic model adjusted by center, as random effect; age; and type of KPC-Kp infection, when appropriate. } \\
\text { tMajor surgery includes any invasive operative procedure in which a more extensive resection is performed, including a body cavity is entered, organs } \\
\text { are removed, or normal anatomy is altered. } \\
\text { §Severe infection included bloodstream or lower respiratory tract infection plus septic shock from other sites; Mild infection included infections from other } \\
\text { sites; and colonized patients were identified through surveillance protocols. }\end{array}$} \\
\hline
\end{tabular}

extended to post-acute care, long-term care, or rehabilitation facilities to control the spread of KPC-Kp. As highlighted by a recent report from the European Centre for Disease Prevention and Control (16), standardized actions for CRE containment in Italy must be driven by comprehensive coordinated responses implemented nationally rather than current practice of delegating responsibilities to the regional or hospital level.

In our setting, the KPC-Kp epidemic appears to be driven by the expansion of 3 major K. pneumoniae clonal lineages, specifically ST307, ST101, and ST258/ ST512. Those epidemic clones have been associated with outbreaks and are reported to have an increased capacity to acquire drug resistance (17-19). Clone ST512 was widely distributed across the centers in our study, confirming its spread in Italy (20). We noted clone ST307 in smaller, scattered clusters but did not note differences in infection severity or death between clones.

We examined the KPC-Kp-associated mortality rate and noted it was highest among patients with severe infections, particularly bloodstream infections with septic shock, which is consistent with previous research (21-25). We found no excess risk for death among patients with mild infection. KPC-Kp often is found in vulnerable hospital populations at high risk for illness and death $(21,26)$. To estimate the effect of KPC-Kp infection on hospital mortality rates, we compared patients with severe and mild infections with colonized patients. Colonized patients who did not have infectious events during hospitalization represented the best available control group because they were hospitalized in the same hospitals at the same time as KPC- Kp infected cases and are known to have similar clinical characteristics and underlying conditions (27).

Regarding therapeutic approaches, we found the initial empirical selection of antimicrobial drug treatment was more frequently adequate in patients with a known previous KPC-Kp colonization. This result is in line with other published studies reporting that for patients with no history of previous colonization, adequate antimicrobial treatment can only be started once the susceptibility profile has been received, and this delay might lead to unfavorable outcomes (28-31). Thus, in geographic regions with high CRE prevalence, extending rectal swab 
specimen surveillance to a broader at-risk hospital population is crucial to reduce time to adequate antimicrobial therapy and, ultimately, to improve patients' outcomes. As previously observed $(4,29)$, a combination of $\geq 2$ active agents have been prescribed predominantly in patients with severe infections and at higher risk for death. Of note, we observed a substantial use of colistin despite its unknown efficacy and poor safety profile (mainly related to renal failure), as documented in other studies (32-34). In addition, ceftazidime/avibactam use has increased since 2018, when it became available for routine clinical use in Italy. However, the use of ceftazidime/ avibactam in nonbacteremic infections should be discouraged to reduce chances of acquired in vitro resistance (35-37). The wide variety of therapeutic regimens, >30 combinations reported in our centers, confirms the need for multicenter randomized trials to identify the most effective combination and dosage of antimicrobial agents.

The major strengths of our study are the size of the sample and the representation of KPC-Kp patients included with homogeneous methodology through an independent network of Lombardy hospitals of different size. The results reveal the multifaceted reality of KPC-Kp infection in clinical settings.

The first limitation of our study is that we focused on the most clinically relevant episode for each patient. Therefore, patients who had a colonization followed by an infection were considered and classified according to this second more severe event only. However, in our setting, this subgroup included only $8 \%$ of the colonized patients. Second, we limited our attention to KPC-Kp strains, ignoring E. coli and other carbapenemase, such as oxacillinase 48-like and New Delhi metallo- $\beta$-lactamase. Nevertheless, the estimated ratio of $K$. pneumoniae to E. coli was 11:1 in Italy (16), and KPC is the only endemic mechanism demonstrating carbapenem resistance (9). Third, despite the inclusion of a large number of infected patients, the multitude of treatment patterns prevented reliable exploration of effects of treatment on clinical outcomes, but the description of this heterogeneity remains one of the findings of this study. Finally, we focused on overall rather than disease-specific mortality rates because we aimed to give a global picture of KPC-Kp burden in the Lombardy region. Cause-specific mortality analysis would have required detailed information on the procedures performed before the events occurring during hospitalization, which was beyond the scope of this study.

In conclusion, our study describes $\mathrm{KPC}-\mathrm{K} p$ in a single region of Italy where KPC-Kp has been endemic since 2013. The KPC-Kp epidemic appears to be driven by the expansion of only 3 major clonal lineages. Therefore, the wide heterogeneity in the proportion and incidence of KPC-Kp infections are presumably largely influenced by surveillance protocols and hospital policies. Consequently, to reverse this trend, Italy needs a strengthened collaborative surveillance system that includes regional plans and strong, centrally coordinated activities at the national level. Furthermore, the wide range of treatments adopted by healthcare facilities in this study highlights the urgent need to accompany the surveillance system with a concerted, aggressive, and prompt antimicrobial stewardship plan.

KPC-Kp Study Group collaborators: Simone Battaglia and Giovanni Lorenzin (IRCCS San Raffaele Scientific Institute, Milan, Italy); Annalisa Cavallero (S. Gerardo de'Tintori Hospital, Monza, Italy); Maddalena Casana and Daria Pocaterra (Humanitas Clinical and Research Centre, Milan); Andrea Cona (San Paolo Hospital, Milan); Antonella D'Arminio Monforte (University of Milan San Paolo Hospital, Milan); Gioconda Brigante and Antonella Carducci (Busto Arsizio Hospital, Busto Arsizio, Italy); Daniela Dalla Gasperina and C. Rovelli (University of Insubria, ASST Sette Laghi-Varese, Italy); Matteo Moro and Paolo Scarpellini (IRCCS San Raffaele Scientific Institute, Milan); Giuliano Rizzardini (ASST Fatebenefratelli Sacco, Milan); Angelo Pan and Alessia Zoncada (Istituti Ospedalieri of Cremona, Cremona, Italy); Marco Franzetti and Alessandro Pandolfo (Ospedale A. Manzoni, Lecco, Italy); Francesco Castelli and Ester Pollastri (University of Brescia and ASST Spedali Civili, Brescia, Italy); Giovanna Travi and Chiara Silvia Vismara (ASST Grande Ospedale Metropolitano Niguarda, Milan); Silvia Garilli (Carlo Poma Hospital, Mantova, Italy); Manuela Piazza and Angelo Regazzetti (Hospital of Lodi, Lodi, Italy); Milena Arghittu and Rosaria Maria Colombo (IRCCS Cà Granda Ospedale Maggiore Policlinico, Milan); Pietro Olivieri (ASST Fatebenefratelli Sacco, Milan); Francesco Petri (University of Milano Bicocca, Monza); and Franca Averara and Francesca Vailati (Papa Giovanni XXIII Hospital, Bergamo, Italy).

\section{Acknowledgments}

We thank Igor Monti for IT support and development of web-based case report forms; Joanne Fleming and Judith D. Baggott for their crucial language editing; Alessandro Soave and Matteo Sironi for graphics support; and Ivana Garimoldi for secretarial assistance. We also thank Alessandra Piatti, Danilo Cereda, and Mariella De Biase from Direzione Generale Welfare-Regione Lombardia for their valuable assistance and support on administrative and regulatory procedures during the project. 
This work was supported by the Italian Ministry of HealthLombardy Region (grant no. RF-2011-02351728). The funder had no role in study design, data collection, analysis, interpretation of the results and writing the report.

M.R., L.C., and A.G. designed the study and obtained funding. M.R. supervised the study conduction. L.C. supervised the statistical analysis. T.I. was responsible for conduction of data collection. D.C. was responsible for preparation and storage of all samples. P.M., S.B., C.A., R.M., P.A.G., C.D.C., S.P., S.G.R., P.B., P. Bonfanti, E.V.H., M.P., G.G., and C.C. collected data. F.G. and D.M.C. performed and analyzed the whole genome sequencing of all samples collected. L.C., I.S., and A.D.A. performed statistical data analysis. The paper was written by M.R., L.C., D.M.C., and A.G. and critically revised by G.N., M.C.R., and A.B. All authors reviewed and approved the final version of the manuscript before submission. The KPC-Kp Study Group contributed substantially to design the data collection form, to reach a shared infection criteria definition, and to enroll all the patients included in the study.

The de-identified patient data used for the results reported in this article, including data in text, tables, figures, and appendices, will be shared along with the study protocol. Data will be available from 3 months to 5 years after article publication. Data will be available to researchers who provide a methodologically sound proposal to achieve their aims. Proposals should be addressed to marianna. rossi@asst-monza.it. To gain access, data applicants will need to sign a data access agreement.

\section{About the Author}

Dr. Rossi is an infectious disease specialist at S. Gerardo Hospital, Monza, Italy. Her primary research interests include antimicrobial resistant organisms, hospital acquired infections and infectious disease surveillance.

\section{References}

1. van Duin D, Doi Y. The global epidemiology of carbapenemase-producing Enterobacteriaceae. Virulence. 2017; 8:460-9. https://doi.org/10.1080/21505594.2016.1222343

2. Nordmann P, Cuzon G, Naas T. The real threat of Klebsiella pneumoniae carbapenemase-producing bacteria. Lancet Infect Dis. 2009;9:228-36. https:/ / doi.org/10.1016/ S1473-3099(09)70054-4

3. Gupta N, Limbago BM, Patel JB, Kallen AJ. Carbapenemresistant Enterobacteriaceae: epidemiology and prevention. Clin Infect Dis. 2011;53:60-7. https:/ / doi.org/10.1093/cid/ cir202

4. Tumbarello M, Viale P, Viscoli C, Trecarichi EM, Tumietto F, Marchese A, et al. Predictors of mortality in bloodstream infections caused by Klebsiella pneumoniae carbapenemaseproducing K. pneumoniae: importance of combination therapy. Clin Infect Dis. 2012;55:943-50. https:/ / doi.org/10.1093/cid/ cis588
5. Stewardson AJ, Marimuthu K, Sengupta S, Allignol A, ElBouseary M, Carvalho MJ, et al. Effect of carbapenem resistance on outcomes of bloodstream infection caused by Enterobacteriaceae in low-income and middle-income countries (PANORAMA): a multinational prospective cohort study. Lancet Infect Dis. 2019;19:601-10. https://doi.org/10.1016/S1473-3099(18)30792-8

6. van Duin D, Kaye KS, Neuner EA, Bonomo RA. Carbapenem-resistant Enterobacteriaceae: a review of treatment and outcomes. Diagn Microbiol Infect Dis. 2013;75:115-20. https://doi.org/10.1016/j.diagmicrobio. 2012.11.009

7. Grundmann H, Glasner C, Albiger B, Aanensen DM, Tomlinson CT, Andrasevic AT, et al. European Survey of Carbapenemase-Producing Enterobacteriaceae (EuSCAPE) Working Group. Occurrence of carbapenemase-producing Klebsiella pneumoniae and Escherichia coli in the European survey of carbapenemase-producing Enterobacteriaceae (EuSCAPE): a prospective, multinational study. Lancet Infect Dis. 2017;2:153-63. https://doi.org/10.1016/ S1473-3099(16)30257-2

8. European Centre for Disease Prevention and Control. Antimicrobial resistance surveillance in Europe 2011. Annual Report of the European Antimicrobial Resistance Surveillance Network (EARS-Net). Stockholm: The Centre; 2012 [cited 2020 Dec 19]. https:/ / www.ecdc.europa.eu/en/ publications-data/antimicrobial-resistance-surveillanceeurope-2011

9. European Centre for Disease Prevention and Control. Antimicrobial resistance surveillance in Europe 2016. Annual Report of the European Antimicrobial Resistance Surveillance Network (EARS-Net). Stockholm: The Centre; 2018 [cited 2020 Dec 19]. https:/ / www.ecdc.europa.eu/en/ publications-data/antimicrobial-resistance-surveillanceeurope-2016

10. Higher Institute of Health. Ar-Iss, antibiotic resistance surveillance in Italy: 2012-2016 data [in Italian] [cited 2020 Dec 19]. https://www.epicentro.iss.it/resistenza_ antibiotici/dati-2012-2016-ar-iss

11. Brolund A, Lagerqvist N, Byfors S, Struelens MJ, Monnet DL, Albiger B, et al.; European Antimicrobial Resistance Genes Surveillance Network EURGen-Net Capacity Survey Group. Worsening epidemiological situation of carbapenemaseproducing Enterobacteriaceae in Europe, assessment by national experts from 37 countries, July 2018. Euro Surveill. 2019;24:24. https:/ / doi.org/10.2807/1560-7917. ES.2019.24.9.1900123

12. Marimuthu K, Venkatachalam I, Khong WX, Koh TH, Cherng BPZ, Van La M, et al.; Carbapenemase-Producing Enterobacteriaceae in Singapore (CaPES) Study Group. Clinical and molecular epidemiology of carbapenemresistant Enterobacteriaceae among adult inpatients in Singapore. Clin Infect Dis. 2017;64(suppl_2):S68-75. https://doi.org/10.1093/cid/cix113

13. Centers for Disease Control and Prevention. CDC/ NHSN surveillance definitions for specific types of infections. Atlanta: The Centers; 2014 [cited 2020 Dec 19]. http:/ / www.socinorte.com/wp-content/ uploads/2014/06/17pscNosInfDef_current.pdf

14. McKinnell JA, Dwyer JP, Talbot GH, Connolly LE, Friedland I, Smith A, et al.; CARE Study Group. Plazomicin for infections caused by carbapenem-resistant Enterobacteriaceae. N Engl J Med. 2019;380:791-3. https:/ / doi.org/10.1056/ NEJMc1807634

15. Clinical and Laboratory Standards Institute. Performance standards for antimicrobial susceptibility testing; 
twenty-fourth informational supplement. Wayne (PA): The Institute; 2014 [cited 2020 Dec 19]. https:/ / webstore. ansi.org/Standards/CLSI/CLSIM100S24

16. European Centre for Disease Prevention and Control. ECDC country visit to Italy to discuss antimicrobial resistance issues. Stockholm: The Centre; 2017 [cited 2020 Dec 19]. https:/ / www.ecdc.europa.eu/en/publications-data/ecdc-countryvisit-italy-discuss-antimicrobial-resistance-issues

17. Navon-Venezia S, Kondratyeva K, Carattoli A. Klebsiella pneumoniae: a major worldwide source and shuttle for antibiotic resistance. FEMS Microbiol Rev. 2017;41:252-75. https://doi.org/10.1093/femsre/fux013

18. Snitkin ES, Zelazny AM, Thomas PJ, Stock F, Henderson DK, Palmore TN, et al.; NISC Comparative Sequencing Program. Tracking a hospital outbreak of carbapenem-resistant Klebsiella pneumoniae with whole-genome sequencing. Sci Transl Med. 2012;4:148ra116. https:/ / doi.org/10.1126/ scitranslmed.3004129

19. Mathers AJ, Peirano G, Pitout JD. The role of epidemic resistance plasmids and international high-risk clones in the spread of multidrug-resistant Enterobacteriaceae. Clin Microbiol Rev. 2015;28:565-91. https:/ / doi.org/10.1128/ CMR.00116-14

20. Conte V, Monaco M, Giani T, D'Ancona F, Moro ML, Arena F, et al.; AR-ISS Study Group on CarbapenemaseProducing K. pneumoniae. Molecular epidemiology of KPC-producing Klebsiella pneumoniae from invasive infections in Italy: increasing diversity with predominance of the ST512 clade II sublineage. J Antimicrob Chemother. 2016;71: 3386-91. https:/ / doi.org/10.1093/jac/dkw337

21. Tumbarello M, Trecarichi EM, De Rosa FG, Giannella M, Giacobbe DR, Bassetti M, et al.; ISGRI-SITA (Italian Study Group on Resistant Infections of the Società Italiana Terapia Antinfettiva). Infections caused by KPC-producing Klebsiella pneumoniae: differences in therapy and mortality in a multicentre study. J Antimicrob Chemother. 2015;70:213343. https://doi.org/10.1093/jac/dkv086

22. Daikos GL, Tsaousi S, Tzouvelekis LS, Anyfantis I, Psichogiou M, Argyropoulou A, et al. Carbapenemaseproducing Klebsiella pneumoniae bloodstream infections: lowering mortality by antibiotic combination schemes and the role of carbapenems. Antimicrob Agents Chemother. 2014;58:2322-8. https:// doi.org/10.1128/AAC.02166-13

23. Patel G, Huprikar S, Factor SH, Jenkins SG, Calfee DP. Outcomes of carbapenem-resistant Klebsiella pneumoniae infection and the impact of antimicrobial and adjunctive therapies. Infect Control Hosp Epidemiol. 2008;29:1099-106. https://doi.org/10.1086/592412

24. Fraenkel-Wandel Y, Raveh-Brawer D, Wiener-Well Y, Yinnon AM, Assous MV. Mortality due to bla ${ }_{\mathrm{KPC}}$ Klebsiella pneumoniae bacteraemia. J Antimicrob Chemother. 2016;71:1083-7. https://doi.org/10.1093/jac/dkv414

25. Bertolini G, Nattino G, Tascini C, Poole D, Viaggi B, Carrara G, et al.; GiViTI Steering Committee. Mortality attributable to different Klebsiella susceptibility patterns and to the coverage of empirical antibiotic therapy: a cohort study on patients admitted to the ICU with infection. Intensive Care Med. 2018;44:1709-19. https:/ / doi.org/ 10.1007/s00134-018-5360-0

26. Hauck C, Cober E, Richter SS, Perez F, Salata RA, Kalayjian RC, et al.; Antibacterial Resistance Leadership Group. Spectrum of excess mortality due to carbapenemresistant Klebsiella pneumoniae infections. Clin Microbiol Infect. 2016;22:513-9. https:/ / doi.org/10.1016/j.cmi.2016.01.023

27. Borer A, Saidel-Odes L, Eskira S, Nativ R, Riesenberg K, Livshiz-Riven I, et al. Risk factors for developing clinical infection with carbapenem-resistant Klebsiella pneumoniae in hospital patients initially only colonized with carbapenem-resistant K. pneumoniae. Am J Infect Control. 2012;40:421-5. https:// doi.org/10.1016/j.ajic.2011.05.022

28. Shimasaki T, Seekatz A, Bassis C, Rhee Y, Yelin RD, Fogg L, et al.; Centers for Disease Control and Prevention Epicenters Program. Increased relative abundance of Klebsiella pneumoniae carbapenemase-producing Klebsiella pneumoniae within the gut microbiota is associated with risk of bloodstream infection in long-term acute care hospital patients. Clin Infect Dis. 2019;68:2053-9. https://doi.org/ 10.1093/cid/ciy796

29. Gutiérrez-Gutiérrez B, Salamanca E, de Cueto M, Hsueh PR, Viale P, Paño-Pardo JR, et al.; REIPI/ESGBIS/INCREMENT Investigators. Effect of appropriate combination therapy on mortality of patients with bloodstream infections due to carbapenemase-producing Enterobacteriaceae (INCREMENT): a retrospective cohort study. Lancet Infect Dis. 2017;17:72634. https:/ / doi.org/10.1016/S1473-3099(17)30228-1

30. Cano A, Gutiérrez-Gutiérrez B, Machuca I, Gracia-Ahufinger I, Pérez-Nadales E, Causse M, et al. Risks of infection and mortality among patients colonized with Klebsiella pneumoniae carbapenemase-producing K. pneumoniae: validation of scores and proposal for management. Clin Infect Dis. 2018;66:1204-10. https:/ / doi.org/10.1093/cid/cix991

31. Giannella M, Trecarichi EM, De Rosa FG, Del Bono V, Bassetti M, Lewis RE, et al. Risk factors for carbapenemresistant Klebsiella pneumoniae bloodstream infection among rectal carriers: a prospective observational multicentre study. Clin Microbiol Infect. 2014;20:1357-62. https:// doi.org/10.1111/1469-0691.12747

32. Giacobbe DR, di Masi A, Leboffe L, Del Bono V, Rossi M, Cappiello D, et al. Hypoalbuminemia as a predictor of acute kidney injury during colistin treatment. Sci Rep. 2018;8:11968. https://doi.org/10.1038/s41598-018-30361-5

33. van Duin D, Lok JJ, Earley M, Cober E, Richter SS, Perez F, et al.; Antibacterial Resistance Leadership Group. Colistin versus ceftazidime-avibactam in the treatment of infections due to carbapenem-resistant Enterobacteriaceae. Clin Infect Dis. 2018;66:163-71. https:// doi.org/10.1093/cid/cix783

34. Perez F, Bonomo RA. Carbapenem-resistant Enterobacteriaceae: global action required. Lancet Infect Dis. 2019;19:561-2. https://doi.org/10.1016/S1473-3099(19)30210-5

35. Shields RK, Potoski BA, Haidar G, Hao B, Doi Y, Chen L, et al. Clinical outcomes, drug toxicity, and emergence of ceftazidime-avibactam resistance among patients treated for carbapenem-resistant Enterobacteriaceae infections. Clin Infect Dis. 2016;63:1615-8. https://doi.org/10.1093/cid/ciw636

36. Shields RK, Nguyen MH, Chen L, Press EG, Kreiswirth BN, Clancy CJ. Pneumonia and renal replacement therapy are risk factors for ceftazidime-avibactam treatment failures and resistance among patients with carbapenem-resistant Enterobacteriaceae infections. Antimicrob Agents Chemother. 2018;62:e02497-17. https:// doi.org/10.1128/AAC.02497-17

37. Tumbarello M, Trecarichi EM, Corona A, De Rosa FG, Bassetti M, Mussini C, et al. Efficacy of ceftazidimeavibactam salvage therapy in patients with infections caused by Klebsiella pneumoniae carbapenemase-producing $K$. pneumoniae. Clin Infect Dis. 2019;68:355-64. https:/ / doi.org/ 10.1093/cid/ciy492

Address for correspondence: Andrea Gori, Infectious Diseases

Unit, Department of Internal Medicine, Fondazione IRCCS Ca' Granda Ospedale Maggiore Policlinico, Padiglione Granelli, first floor, Via Sforza 35 Milan 20122, Italy; email: andrea.gori@unimi.it 